\title{
Chern-Simons term at finite density
}

\author{
A.N.Sissakian ${ }^{a}$, O.Yu.Shevchenko ${ }^{b}$, and S.B.Solganik ${ }^{b}$, f \\ ${ }^{a}$ Bogolubov Laboratory of Theoretical Physics, Joint Institute for Nuclear Research, Dubna, \\ Moscow region 141980, Russia \\ ${ }^{b}$ Laboratory of Nuclear Problems, Joint Institute for Nuclear Research, Dubna, Moscow region \\ 141980, Russia
}

\begin{abstract}
The Chern-Simons topological term coefficient is derived at arbitrary finite density. As it occures that $\mu^{2}=m^{2}$ is the crucial point for Chern-Simons. So when $\mu^{2}<m^{2} \mu-$ influence disappears and we get the usual Chern-Simons term. On the other hand when $\mu^{2}>m^{2}$ the Chern-Simons term vanishes because of non-zero density of background fermions. In particular for massless case parity anomaly is absent at any finite density. This result holds in any odd dimension as in abelian so as in nonabelian cases.
\end{abstract}

PACS: 11.15.-q, 05.30.Fk, 11.30.Er

The great number of papers devoted to the Chern-Simons topological term since [1] and by now have appeared. This interest is based on variety of significant physical effects caused by Chern-Simons secondary characteristic class. These are, for example, gauge particles mass generation in quantum field theory, applications to condense matter physics such as the fractional quantum Hall effect and high $T_{c}$ superconductivity, possibility of free of metric tensor theory construction and so on.

In a conventional zero density gauge theory it was shown [2-4] that the Chern-Simons term is generated in the Eulier-Heisenberg effective action by quantum corrections. The main goal of this paper is to explore the dynamical generation of the parity anomalous Chern-Simons term at finite density in odd dimensional gauge theory.

In the excellent paper by Niemi [5] it was emphasized that the charge density at $\mu \neq 0$ becomes nontopological object, i.e contains as topological part so as nontopological one. The charge density at $\mu \neq 0$ (nontopological, neither parity odd nor parity even object) $]$ in $Q E D_{3}$ at finite density was calculated and exploited in [G]. Here we are interested in effect of finite density influence on covariant parity odd form in action leading to the gauge field mass generation Chern-Simons topological term. Deep insight on this phenomena at small densities was done in [5, [7]. The result for Chern-Simons term coefficient in $Q E D_{3}$ is $[\operatorname{sgn}(m-\mu)+\operatorname{sgn}(m+\mu)]$ (see [7], formulas (10.19) ). However, to get this result it was heuristicaly supposed that at small densities index theorem could still be used and only odd in energy part of spectral density is responsible for parity nonconserving effect. Because of this in [7] it had been stressed that the result holds only for small $\mu$. However, as we'll see below this result holds for any values of chemical potential. Thus, to obtain trustful result at any values of $\mu$ one have to use transparent and free of any restrictions on $\mu$ procedure, which would allow to perform calculations with arbitrary nonabelian background gauge fields. That is why, we will use

\footnotetext{
${ }^{1}$ E-mail address: shevch@nusun.jinr.dubna.su

${ }^{2}$ E-mail address: solganik@thsun1.jinr.dubna.su

${ }^{3}$ For abbreviation, speaking about parity invariance properties of local objects, we will keep in mind symmetries of the corresponding action parts.
} 
here perturbative technique for Chern-Simons term calculation. In addition this approach is completely covariant.

Since the chemical potential term $\mu \bar{\psi} \gamma^{0} \psi$ is odd under charge conjugation we can expect that it would contribute to $P$ and $C P$ nonconserving quantity - Chern-Simons term. As we will see, this expectation is completely justified.

The zero density approach usually is a good quantum field approximation when value of chemical potential is small as compared with characteristic energy scale of physical processes. Nevertheless, for investigation of topological effects it is not the case. As we will see below, even small density presence could lead to the principal effects.

Introduction of a chemical potential $\mu$ in a theory corresponds to the presence of a nonvanishing background fermion density. It must be emphasized that the formal addition of a chemical potential looks like a simple gauge transformation with the gauge function $\mu t$. However, it doesn't only shift the time component of a vector potential but also gives corresponding prescription for handling Green's function poles. The correct introduction of a chemical potential redefines the ground state (Fermi energy), which leads to a new spinor propagator with the proper $\epsilon$-prescription for poles. So, for the free spinor propagator we have (see, for example, 8, 9])

$$
S(p ; \mu)=\frac{\tilde{\not}+m}{\left(\tilde{p_{0}}+i \epsilon \operatorname{sgn} p_{0}\right)^{2}-\vec{p}^{2}-m^{2}},
$$

where $\tilde{p}=\left(p_{0}+\mu, \vec{p}\right)$. It is easy to see that in $\mu=0$ case one at once gets the usual $\epsilon$-prescription because of the positivity of $p_{0} \operatorname{sgn} p_{0}$.

Let's first consider nonabelian 3-dimensional gauge theory. The only graphs whose P-odd parts contribute to the parity anomalous Chern-Simons term are shown in Fig.1.

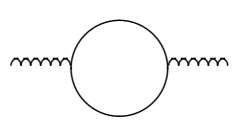

(a)

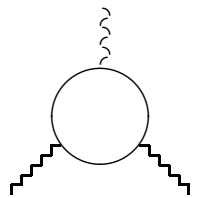

(b)

Fig.1 Graphs whose P-odd parts contribute to the Chern-Simons term in nonabelian 3D gauge theory

So, the part of effective action containing the Chern-Simons term looks as

$$
\begin{aligned}
I_{\text {eff }}^{C . S .} & =\frac{1}{2} \int_{x} A_{\mu}(x) \int_{p} \mathrm{e}^{-i x p} A_{\nu}(p) \Pi^{\mu \nu}(p) \\
& +\frac{1}{3} \int_{x} A_{\mu}(x) \int_{p, r} \mathrm{e}^{-i x(p+r)} A_{\nu}(p) A_{\alpha}(r) \Pi^{\mu \nu \alpha}(p, r),
\end{aligned}
$$

where polarization operator and vertice have a standard form

$$
\begin{aligned}
\Pi^{\mu \nu}(p) & =g^{2} \int_{k} \operatorname{tr}\left[\gamma^{\mu} S(p+k ; \mu) \gamma^{\nu} S(k ; \mu)\right] \\
\Pi^{\mu \nu \alpha}(p, r) & =g^{3} \int_{k} \operatorname{tr}\left[\gamma^{\mu} S(p+r+k ; \mu) \gamma^{\nu} S(r+k ; \mu) \gamma^{\alpha} S(k ; \mu) .\right] .
\end{aligned}
$$


First consider the second order term (Fig.1, graph (a)). It is well-known that the only object giving us the possibility to construct $P$ and $T$ odd form in action is Levi-Chivita tensor . $^{\text {. }}$ Thus, we will drop all terms noncontaining Levi-Chivita tensor. Signal for the mass generation (Chern-Simons term) is $\Pi^{\mu \nu}\left(p^{2}=0\right) \neq 0$. So we get

$$
\Pi^{\mu \nu}=g^{2} \int_{k}\left(-i 2 m e^{\mu \nu \alpha} p_{\alpha}\right) \frac{1}{\left(\tilde{k}^{2}-m^{2}+i \epsilon\left(k_{0}+\mu\right) \operatorname{sgn}\left(k_{0}\right)\right)^{2}} .
$$

After some simple algebra one obtains

$$
\begin{aligned}
\Pi^{\mu \nu} & =-i 2 m g^{2} e^{\mu \nu \alpha} p_{\alpha}\left[\int \frac{d^{3} k}{(2 \pi)^{3}} \frac{1}{\left(\tilde{k}^{2}-m^{2}+i \epsilon\right)^{2}}\right. \\
& \left.-\int \frac{d k_{0}}{2 \pi} \theta\left(-\left(k_{0}+\mu\right) \operatorname{sgn}\left(k_{0}\right)\right) \int \frac{d^{2} k}{(2 \pi)^{2}}\left(\frac{1}{\left(\tilde{k}^{2}-m^{2}+i \epsilon\right)^{2}}-\frac{1}{\left(\tilde{k}^{2}-m^{2}-i \epsilon\right)^{2}}\right)\right] .
\end{aligned}
$$

Using the well-known identity

$$
\Im m \frac{1}{a \pm i \varepsilon}=\mp i \pi \delta(a)
$$

and taking over integrals one immediately comes to

$$
\Pi^{\mu \nu}=-i \frac{m}{|m|} \frac{g^{2}}{4 \pi} e^{\mu \nu \alpha} p_{\alpha} \theta\left(m^{2}-\mu^{2}\right) .
$$

In the same manner handling the third order contribution (Fig.1b) one gets

$$
\begin{aligned}
\Pi^{\mu \nu \alpha} & =-2 g^{3} i e^{\mu \nu \alpha} \int \frac{d^{3} k}{(2 \pi)^{3}}\left[\frac{m\left(k^{2}-m^{2}\right)}{\left(k^{2}-m^{2}+i \varepsilon \operatorname{sgn}\left(k_{0}\right)\left(k_{0}+\mu\right)\right)^{3}}\right] \\
& =-i 2 m g^{3} e^{\mu \nu \alpha} \int \frac{d^{3} k}{(2 \pi)^{3}}\left[\frac{1}{\left(k^{2}-m^{2}+i \varepsilon \operatorname{sgn}\left(k_{0}\right)\left(k_{0}+\mu\right)\right)^{2}}\right]
\end{aligned}
$$

and further all calculations are identical to the second order

$$
\Pi^{\mu \nu \alpha}=-i \frac{m}{|m|} \frac{g^{3}}{4 \pi} e^{\mu \nu \alpha} \theta\left(m^{2}-\mu^{2}\right) .
$$

Substituting (6), (8) in the effective action (2) we get eventually

$$
I_{\text {eff }}^{C . S .}=\frac{m}{|m|} \theta\left(m^{2}-\mu^{2}\right) \frac{g^{2}}{8 \pi} \int d^{3} x e^{\mu \nu \alpha} \operatorname{tr}\left(A_{\mu} \partial_{\nu} A_{\alpha}-\frac{2}{3} g A_{\mu} A_{\nu} A_{\alpha}\right) .
$$

Thus, we get Chern-Simons term with $\mu$ dependent coefficient.

Let's now consider 5-dimensional gauge theory. Here the Levi-Chivita tensor is 5-dimensional $e^{\mu \nu \alpha \beta \gamma}$ and the relevant graphs are shown in Fig.2.

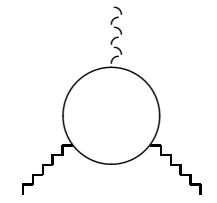

(a)

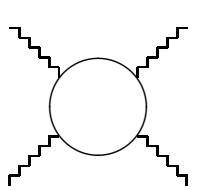

(b)

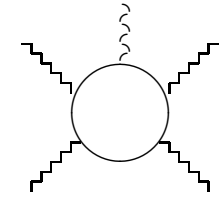

(c)

Fig.2 Graphs whose P-odd parts contribute to the Chern-Simons term in nonabelian 5D theory

\footnotetext{
${ }^{4}$ In three dimensions it arises as a trace of three $\gamma$-matrices (Pauli matrices)
} 
The part of effective action containing Chern-Simons term reads

$$
\begin{aligned}
I_{\text {eff }}^{C . S .} & =\frac{1}{3} \int_{x} A_{\mu}(x) \int_{p, r} \mathrm{e}^{-i x(p+r)} A_{\nu}(p) A_{\alpha}(r) \Pi^{\mu \nu \alpha}(p, r) \\
& +\frac{1}{4} \int_{x} A_{\mu}(x) \int_{p, r} \mathrm{e}^{-i x(p+r+s)} A_{\nu}(p) A_{\alpha}(r) A_{\beta}(s) \Pi^{\mu \nu \alpha \beta}(p, r, s) \\
& +\frac{1}{5} \int_{x} A_{\mu}(x) \int_{p, r} \mathrm{e}^{-i x(p+r+s+q)} A_{\nu}(p) A_{\alpha}(r) A_{\beta}(s) A_{\gamma}(s) \Pi^{\mu \nu \alpha \beta \gamma}(p, r, s, q)
\end{aligned}
$$

All calculations are similar to 3-dimensional case. First consider third order contribution (Fig.2a)

$$
\Pi^{\mu \nu \alpha}(p, r)=g^{3} \int_{k} \operatorname{tr}\left[\gamma^{\mu} S(p+r+k ; \mu) \gamma^{\nu} S(r+k ; \mu) \gamma^{\alpha} S(k ; \mu)\right] .
$$

Taking into account that trace of five $\gamma$-matrices in 5 -dimensions is

$$
\operatorname{tr}\left[\gamma^{\mu} \gamma^{\nu} \gamma^{\alpha} \gamma^{\beta} \gamma^{\rho}\right]=4 i e^{\mu \nu \alpha \beta \rho},
$$

we extract the parity odd part of the vertice

$$
\Pi^{\mu \nu \alpha}=g^{3} \int \frac{d^{5} k}{(2 \pi)^{5}}\left(i 4 m e^{\mu \nu \alpha \beta \sigma} p_{\beta} r_{\sigma}\right) \frac{1}{\left(\tilde{k}^{2}-m^{2}+i \epsilon\left(k_{0}+\mu\right) \operatorname{sgn}\left(k_{0}\right)\right)^{3}},
$$

or in more transparent way

$$
\begin{aligned}
\Pi^{\mu \nu \alpha} & =i 4 m g^{3} e^{\mu \nu \alpha \beta \sigma} p_{\alpha} r_{\sigma}\left[\int \frac{d^{5} k}{(2 \pi)^{5}} \frac{1}{\left(\tilde{k}^{2}-m^{2}+i \epsilon\right)^{3}}\right. \\
& \left.-\int \frac{d k_{0}}{2 \pi} \theta\left(-\left(k_{0}+\mu\right) \operatorname{sgn}\left(k_{0}\right)\right) \int \frac{d^{4} k}{(2 \pi)^{4}}\left(\frac{1}{\left(\tilde{k}^{2}-m^{2}+i \epsilon\right)^{3}}-\frac{1}{\left(\tilde{k}^{2}-m^{2}-i \epsilon\right)^{3}}\right)\right] .
\end{aligned}
$$

Evaluating integrals one comes to

$$
\Pi^{\mu \nu \alpha}=i \frac{m}{|m|} \frac{g^{3}}{16 \pi^{2}} e^{\mu \nu \alpha \beta \sigma} p_{\alpha} r_{\sigma} \theta\left(m^{2}-\mu^{2}\right) .
$$

In the same way operating graphs (b) and (c) (Fig.2) one will obtain

$$
\Pi^{\mu \nu \alpha \beta}=i \frac{m}{|m|} \frac{g^{4}}{8 \pi^{2}} e^{\mu \nu \alpha \beta \sigma} s_{\sigma} \theta\left(m^{2}-\mu^{2}\right)
$$

and

$$
\Pi^{\mu \nu \alpha \beta \gamma}=i \frac{m}{|m|} \frac{g^{5}}{16 \pi^{2}} e^{\mu \nu \alpha \beta \sigma} \theta\left(m^{2}-\mu^{2}\right) .
$$

Substituting (14) - (16) in the effective action (10) we get the final result for Chern-Simons in 5-dimensional theory

$$
\begin{aligned}
I_{\text {eff }}^{C . S .} & =\frac{m}{|m|} \theta\left(m^{2}-\mu^{2}\right) \frac{g^{3}}{48 \pi^{2}} \int d^{5} x e^{\mu \nu \alpha \beta \gamma} \\
& \times \operatorname{tr}\left(A_{\mu} \partial_{\nu} A_{\alpha} \partial_{\beta} A_{\gamma}+\frac{3}{2} g A_{\mu} A_{\nu} A_{\alpha} \partial_{\beta} A_{\gamma}+\frac{3}{5} g^{2} A_{\mu} A_{\nu} A_{\alpha} A_{\beta} A_{\gamma}\right) .
\end{aligned}
$$


It is remarkable that all parity odd contributions are finite as in 3-dimensional so as in 5-dimensional cases. Thus, all values in the effective action are renormalized in a standard way, i.e. the renormalizations are determined by conventional (parity even) parts of vertices.

From the above direct calculations it is clearly seen that the chemical potential dependent coefficient is the same for all parity odd parts of diagrams and doesn't depend on space dimension. So, the influence of finite density on Chern-Simons term generation is the same in any odd dimension:

$$
I_{e f f}^{\mathrm{C} . \mathrm{S}}=\frac{m}{|m|} \theta\left(m^{2}-\mu^{2}\right) \pi W[A],
$$

where $W[A]$ is the Chern-Simons secondary characteristic class in any odd dimension. Since only the lowest orders of perturbative series contribute to CS term at finite density (the same situation is well-known at zero density), the result obtained by using formally perturbative technique appears to be nonperturbative. Thus, the $\mu$-dependent CS term coefficient reveals the amazing property of universality. Namely, it does depend on neither dimension of the theory nor abelian or nonabelian gauge theory is studied.

The arbitrariness of $\mu$ gives us the possibility to see Chern-Simons coefficient behavior at any masses. It is very interesting that $\mu^{2}=m^{2}$ is the crucial point for Chern-Simons. Indeed,

it is clearly seen from (18) that when $\mu^{2}<m^{2} \mu$-influence disappears and we get the usual Chern-Simons term $I_{e f f}^{\text {C.S }}=\pi W[A]$. On the other hand when $\mu^{2}>m^{2}$ the situation is absolutely different. One can see that here the Chern-Simons term disappears because of non-zero density of background fermions. We'd like to emphasize the important massless case $m=0$ considered in [7]. Then even negligible density, which always take place in any physical processes, leads to vanishing of the parity anomaly.

In conclusion, let us stress again that we nowhere have used any restrictions on $\mu$. Thus we not only confirm result in [7] for Chern-Simons in $Q E D_{3}$ at small density, but also expand it on arbitrary $\mu$, nonabelian case and arbitrary odd dimension.

\section{References}

[1] R. Jackiw, S. Templeton Phys.Rev. D23 (1981) 2291.

[2] A. J. Niemi and G. W. Semenoff Phys.Rev.Lett. 51 (1983) 2077.

[3] A. N. Redlich Phys.Rev. D29 (1984) 2366.

[4] L. Alvarez-Gaume, E. Witten Nucl.Phys. B234 (1984) 269.

[5] A. J. Niemi Nucl.Phys. B251[FS13] (1985) 155.

[6] J. D. Lykken, J. Sonnenschen and N. Weiss Phys.Rev D42 (1990) 2161; Int.J.Mod.Phys. A6 (1991) 5155 ; A. M. J. Schakel Phys.Rev. D43 (1991) 1428; V. Y. Zeitlin Mod.Phys.Lett. A8 (1993) 1821.

[7] A. J. Niemi and G. W. Semenoff Phys.Rep. 135 No.3 (1986) 99.

[8] E. V. Shuryak Phys.Rep. 61 (1980) 73.

[9] A. Chodos, K. Everding and D. A. Owen Phys.Rev. D42 (1990) 2881. 